09,08

\title{
Люминесцентные свойства активированных нанокомпозитов на основе наночастиц структуры „ядро-оболочка“
}

\author{
(C) K.K. Пухов \\ Институт общей фризики им. А.М. Прохорова РАН, \\ Москва, Россия \\ E-mail: pukhov@Ist.gpi.ru
}

Выведены формулы для скоростей спонтанного излучательного распада возбуждения люминесцентных центров, находящихся в субволновых сферических наночастицах структуры „ядро-оболочка“ и в матрице нанокомпозита. Обсуждаются модели эффективной среды и проблемы локального поля, связанные с оптическими свойствами активированных нанокомпозитов на основе наночастиц структуры „ядро-оболочка“. Проведен детальный анализ двух современных подходов к проблеме спонтанных переходов в люминесцентных центрах, находящихся в однородных сферических наночастицах.

Работа поддержана Президиумом РАН (Программа 5 „Фотонные технологии и зондирование неоднородных сред и биообъектов“").

DOI: $10.21883 /$ FTT.2019.05.47606.21F

\section{1. Введение}

Люминесцентные свойства наночастиц (НЧ), активированных редкоземельными (Р3) ионами и ионами переходных металлов с возрастающей интенсивностью исследуются последние два десятилетия. Особенно большое внимание уделяется НЧ, активированным Р3-ионами, что обусловлено их уникальными люминесцентными свойствами: узкие спектральные линии, относительно большие времена жизни (в диапазоне 1-100 ms), большие стоксовый и антистоксовый сдвиги, высокий квантовый выход, высокая стойкость к фотообесцвечиванию и низкая токсичность [1-4]. Эти свойства делают их привлекательными для множества разнообразных применений: от биосенсоров до однофотонных источников для квантовой информатики. Они, в частности, рассматриваются как перспективные материалы для преобразования ИК- и УФ-излучения в видимое излучение процессами ап- и даун-конверсии [5-7]. Развитая структура Р3-ионов также позволяет успешно конвертировать излучение используя ступенчатые многофотонные процессы [8-10]. В настоящее время названные процессы конвертирования особенно актуальны, как метод повышения эффективности солнечных элементов на основе НЧ, активированных РЗ-ионами. Являясь перспективными „строительными блоками“ нанотехнологий, НЧ открывают, в том числе, и новые направления создания оригинальных лазерных сред [11]. В 2012 г. реализован лазер на основе коллоидного раствора НЧ $\mathrm{Nd}_{2} \mathrm{O}_{3}$ [12]. Эти среды перспективны для решения проблемы тепловыделения в объеме активных элементов мощных киловаттных лазеров. Отметим также, что активированные Р3-ионами НЧ обладают уникальным термометрическим откликом, что открывает перспективы создания высокочувствительных люминесцентных термометров с наномасштабным пространственным разрешением [13]. Во всех перечисленных примерах существенную, если не определяющую, роль играют излучательные переходы.

Сейчас известно, что излучательные времена жизни $(\tau)$ возбужденных состояний люминесцентных центров (ЛЦ) в НЧ значительно отличаются от таковых в объемных кристаллах того же состава и структуры [1-4,14]. Впервые это экспериментально было показано в работе [14]. Существенно, что при этом была обнаружена зависимость $\tau$ от диэлектрических характеристик среды, заполняющей пространство между НЧ. (Далее везде, если это специально не оговорено, мы под временем жизни $\tau$ будем понимать излучательные времена жизни).

Растущий интерес исследователей вызывают НЧструктуры „ядро-оболочка“, как однослойные [15], так и двуслойные [16]. Появляется широкая возможность целенаправленного выбора параметров оболочки и ядра слоистых частиц для получения желаемых свойств. В этой связи фундаментальное и прикладное значение приобретает проблема установления соотношений между излучательными свойствами ЛЦ и характеристиками нанокомпозита (НК), определяющих, в том числе, и степень изменения люминесцентных характеристик ЛЦ по сравнению с таковыми в однородной среде. (Под термином нанокомпозит здесь и далее понимается диэлектрическая прозрачная среда, содержащая как включения субволновые сферические НЧ, которые, однако, достаточно велики, чтобы могли характеризоваться такими макроскопическими параметрами, как диэлектрическая проницаемость).

Отметим, что современная теория излучательных переходов, созданная на базе квантовой механики и квантовой электродинамики, устанавливает тесную связь между вероятностями спонтанного излучения (коэффициент Эйнштейна), вынужденного излучения и вероятностями переходов, сопровождающихся поглощением излучения [17]. Для теплового излучения соотношения 
между этими вероятностями были впервые найдены Эйнштейном. Зная вероятности спонтанных переходов нетрудно получить вероятности и других переходов.

\section{2. Геометрия задачи. Поля в изолированной НЧ}

Ниже приводятся соотношения между макроскопическими полями в изолированной НЧ и полем внешней электромагнитной волны в матрице, так как эти соотношения понадобятся в дальнейшем. Рассмотрим изолированную субволновую сферическую НЧ-структуры „ядро-оболочка“ (рисунок), находящуюся в поле $\mathbf{E}_{1}$ внешней электромагнитной волны. Под термином „изолированная частица“ здесь и далее понимается НЧ в матрице, в которой расстояние между НЧ намного превышает длину волны в нанокомпозите. А под субволновой частицей здесь и далее подразумевается частица, радиус $(a)$ которой много меньше длины $\lambda$ электромагнитной волны, распространяющейся в композитной среде. Количественно это условие выражается неравенством $k a \ll 1$ для параметра дифракции $k a$, где $k$ - волновое число (здесь и далее мы рассматриваем только композит с вещественными $\varepsilon_{1}, \varepsilon_{2}$, и $\left.\varepsilon_{3}\right)$. Для субволновых частиц справедливо электростатическое приближение и хорошо известно $[18,19]$, что в этом приближении электрическое поле в ядре НЧ однородно, причём его напряженность параллельна напряженности поля в матрице $\mathbf{E}_{1}$ и равна

$$
\mathbf{E}_{3}=\left(9 \varepsilon_{1} \varepsilon_{2} / \Delta\right) \mathbf{E}_{1} .
$$

Знаменатель $\Delta$ формулы (1) определяется выражением $[18,19]$

$$
\Delta=\left(\varepsilon_{2}+2 \varepsilon_{1}\right)\left(\varepsilon_{3}+2 \varepsilon_{2}\right)+2 \xi\left(\varepsilon_{2}-\varepsilon_{1}\right)\left(\varepsilon_{3}-\varepsilon_{2}\right),
$$

где $\xi=(b / a)^{3}$. Электрическое поле внутри оболочки является суперпозицией однородного поля

$$
\mathbf{E}_{2}=\left[3 \varepsilon_{1}\left(\varepsilon_{3}+2 \varepsilon_{2}\right) / \Delta\right] \mathbf{E}_{1}
$$

и поля, эквивалентного полю точечного диполя, расположенного в центре НЧ и имеющего дипольный момент

$$
\mathbf{p}_{2}=\alpha_{2} \mathbf{E}_{1}
$$

где $\alpha_{2}=3 b^{3} \varepsilon_{1}\left(\varepsilon_{3}-\varepsilon_{2}\right) / \Delta$ есть поляризуемость ядра частицы.

Электрическое поле вне частицы является суперпозицией поля $\mathbf{E}_{1}$ и поля, эквивалентного полю точечного диполя, расположенного в центре НЧ и имеющего дипольный момент

$$
\mathbf{p}_{1}=\alpha_{1} \mathbf{E}_{1}
$$

где поляризуемость $\alpha_{1}$ есть поляризуемость всей НЧ, определяемая формулой

$$
\alpha_{1}=a^{3}\left[\left(\varepsilon_{2}-\varepsilon_{1}\right)\left(2 \varepsilon_{1}+\varepsilon_{3}\right)+\xi\left(\varepsilon_{3}-\varepsilon_{2}\right)\left(\varepsilon_{1}+2 \varepsilon_{2}\right)\right] / \Delta .
$$

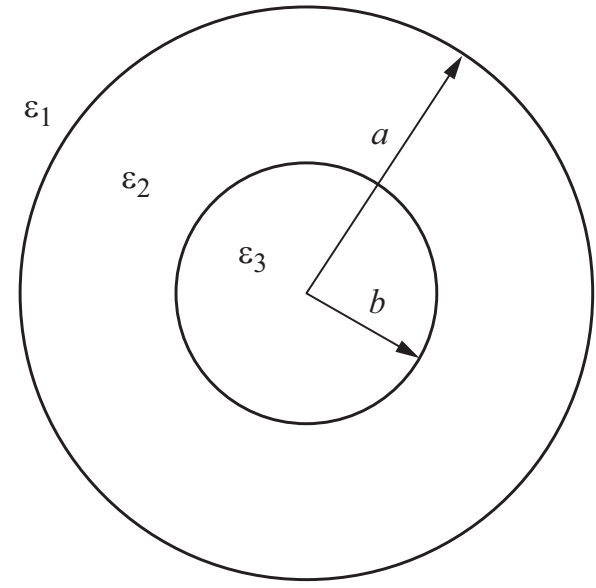

Геометрия задачи: $a-$ радиус всей НЧ, $b-$ радиус ядра НЧ; $\varepsilon_{1}, \varepsilon_{2}$ и $\varepsilon_{3}$ - диэлектрические проницаемости окружающей НЧ среды (матрицы), оболочки и ядра соответственно.

\section{3. Диэлектрическая проницаемость нанокомпозита и поля в НЧ в составе нанокомпозита}

Проблема оптических и, в более широком смысле, электромагнитных свойств гетерогенной среды возникла уже на ранней стадии становления электродинамики сплошных сред. Соответствующий раздел электродинамики сейчас часто называют теорией эффективной среды и главной задачей этой теории является определение диэлектрической (и/или магнитной) проницаемости композитной среды $\varepsilon_{\text {eff }}$ (и/или $\left.\mu_{\text {eff }}\right)$. Наиболее популярна аппроксимация Максвелла Гарнетта [20-24], найденная и апробированная более ста лет назад в работах [20,21]. Максвелл Гарнетт исследовал оптические свойства композита, состоящего из диэлектрической прозрачной матрицы (с диэлектрической проницаемостью $\varepsilon_{\text {med }}$, , содержащей как включения сферические однородные металлические частицы (с диэлектрической проницаемостью $\left.\varepsilon_{\text {spher }}\right)$, и нашел формулу [20,21]

$$
\varepsilon_{\mathrm{eff}} / \varepsilon_{\mathrm{med}}=1+3 f \beta(1-f \beta)
$$

где $f$ есть так называемый фактор заполнения (filling factor) равный объемной доле частиц в композите, а

$$
\beta=\left(\varepsilon_{\text {spher }}-\varepsilon_{\text {med }}\right) /\left(\varepsilon_{\text {spher }}+2 \varepsilon_{\text {med }}\right) .
$$

Заметим, что поляризуемость $\alpha$ шара (с радиусом $a$ ) связана с $\beta$ соотношением $\alpha=a^{3} \beta$, в силу чего (5) можно переписать в виде

$$
\left(\varepsilon_{\mathrm{eff}}-\varepsilon_{\mathrm{med}}\right) /\left(\varepsilon_{\mathrm{eff}}+2 \varepsilon_{\mathrm{med}}\right)=(4 \pi / 3) N_{\text {shpher }} \alpha,
$$

где $N_{\text {shpher }}$ есть число сферических частиц в единице объема композита. Эта форма записи прямо указывает на родство формулы Максвелла Гарнетта с формулами Лорентц-Лоренца и Клаузиуса-Моссотти (см. напр., [25] 
стр. 97, формула (17)). (Необходимым условием применимости формулы Максвелла Гарнетта является уже упомянутое выше неравенство $k a \ll 1$.) Запись формулы Максвелла Гарнетта в форме (6) наводит на мысль, что эта формула будет справедлива и для слоистых сферических частиц. Действительно, правомерность распространения формулы (6) на слоистые сферические частицы доказана в работе [26].

Формула (6) определяет показатель преломления $n_{\text {eff }}=\sqrt{\varepsilon_{\text {eff }}}$ и тем самым устанавливает плотность фотонных состояний в нанокомпозите

$$
\rho_{\mathrm{eff}}(\omega)=n_{\mathrm{eff}}^{3} \omega^{2} / \pi^{2} c^{3} .
$$

Плотность фотонных состояний важный, но не единственный фактор, влияющий на скорость излучательного распада возбуждения ЛЦ [27]. Вторым важным фактором является амплитуда нулевых колебаний, ответственных за спонтанный распад. (Первый упомянутый фактор известен как эффект Парселла [28].) Поэтому необходимы еще соотношения между полем в нанокомпозите $\mathbf{E}$ и полями в матрице, оболочке и ядре. Чтобы найти эти соотношения прибегнем к методу, изложенному в монографии Борена и Хафмена [22].

Определим среднее электрическое поле $\langle\mathbf{E}(\mathbf{r})\rangle$ как поле, усредненное по объему $\tilde{V}$, окружающему точку $\mathbf{r}$, а именно

$$
\langle\mathbf{E}(\mathbf{r})\rangle=(1 / \tilde{V}) \int_{\tilde{V}} \mathbf{E}(\mathbf{r}+\boldsymbol{\rho}) d \boldsymbol{\rho}
$$

Объем $\tilde{V}$ складывается из части объема матрицы около точки $\mathbf{r}$ и объема всех включений в ней. Естественно, объем $\tilde{V}$ должен содержать достаточно большое число включений. Диэлектрическая проницаемость композита $\varepsilon_{\text {eff }}=n_{\text {eff }}^{2}\left(n_{\text {eff }}-\right.$ показатель преломления композита $)$ определяется соотношением [22,29]

$$
\langle\mathbf{D}\rangle=\varepsilon_{\text {eff }}\langle\mathbf{E}\rangle .
$$

Уравнение для средних значений напряженностей электрического поля в нанокомпозите $(\langle\mathbf{E}\rangle)$, матрице $\left(\left\langle\mathbf{E}_{1}\right\rangle\right)$, оболочке $\left(\left\langle\mathbf{E}_{2}\right\rangle\right)$ и ядре $\left(\left\langle\mathbf{E}_{3}\right\rangle\right)$ НЧ имеет вид

$$
\langle\mathbf{E}\rangle=(1-f)\left\langle\mathbf{E}_{1}\right\rangle+f\left[(1-\xi)\left\langle\mathbf{E}_{2}\right\rangle+\xi\left\langle\mathbf{E}_{3}\right\rangle\right] .
$$

Уравнение для средних значений векторов поляризации нанокомпозита $(\langle\mathbf{P}\rangle)$, матрицы $\left(\left\langle\mathbf{P}_{1}\right\rangle\right)$, оболочки $\left(\left\langle\mathbf{P}_{2}\right\rangle\right)$ и ядра $\left(\left\langle\mathbf{P}_{3}\right\rangle\right)$ НЧ дается аналогичной формулой

$$
\langle\mathbf{P}\rangle=(1-f)\left\langle\mathbf{P}_{1}\right\rangle+f\left[(1-\xi)\left\langle\mathbf{P}_{2}\right\rangle+\xi\left\langle\mathbf{P}_{3}\right\rangle\right] .
$$

Примем, следуя [22], что связь между $\left\langle\mathbf{E}_{3}\right\rangle$ и $\left\langle\mathbf{E}_{1}\right\rangle$ в (10) такая же как между $\left\langle\mathbf{E}_{3}\right\rangle$ и $\mathbf{E}_{1}$ в соотношении (1), а связь между $\left\langle\mathbf{E}_{2}\right\rangle$ и $\left\langle\mathbf{E}_{1}\right\rangle$ такая же как между $\left\langle\mathbf{E}_{2}\right\rangle$ и $\left\langle\mathbf{E}_{1}\right\rangle$ в (2).

Тогда уравнение (10) принимает вид

$$
\langle\mathbf{E}\rangle=\left(1-f \beta_{1}\right)\left\langle\mathbf{E}_{1}\right\rangle,
$$

где

$$
\begin{aligned}
\beta_{1} & =\frac{\alpha_{1}}{a^{3}} \\
& =\frac{\left(\varepsilon_{2}-\varepsilon_{1}\right)\left(\varepsilon_{3}+2 \varepsilon_{2}\right)+\xi\left(\varepsilon_{3}-\varepsilon_{2}\right)\left(\varepsilon_{1}+2 \varepsilon_{2}\right)}{\left(\varepsilon_{2}+2 \varepsilon_{1}\right)\left(\varepsilon_{3}+2 \varepsilon_{2}\right)+2 \xi\left(\varepsilon_{2}-\varepsilon_{1}\right)\left(\varepsilon_{3}-\varepsilon_{2}\right)} .
\end{aligned}
$$

Аналогично, из уравнения (11) получаем

$$
\varepsilon_{\mathrm{eff}}\langle\mathbf{E}\rangle=\varepsilon_{1}\left(1+2 \beta_{1} f\right)\left\langle\mathbf{E}_{1}\right\rangle .
$$

Из уравнений (12) и (14) находим выражение для $\varepsilon_{\text {eff: }}$

$$
\varepsilon_{\mathrm{eff}} / \varepsilon_{1}=1+3 f \beta_{1}\left(1-f \beta_{1}\right),
$$

которое совпадает с выражением, вытекающим из общей формулы (6). Формула (15) переходит в формулу Максвелла Гарнетта (4), если в (15) положить $\varepsilon_{2}=\varepsilon_{3}$ или $\xi=(b / a)^{3}=0$. Полученные соотношения для полей запишем в виде

$$
\left\langle\mathbf{E}_{s}\right\rangle=g_{s}\langle\mathbf{E}\rangle,
$$

где

$$
\begin{gathered}
g_{1}=1 /(1-f \beta), \\
g_{2}=3 \varepsilon_{1}\left(\varepsilon_{3}+2 \varepsilon_{2}\right) /[\Delta(1-f \beta)], \\
g_{3}=9 \varepsilon_{1} \varepsilon_{2} /[\Delta(1-f \beta)] .
\end{gathered}
$$

\section{4. Гамильтониан взаимодействия и вероятности переходов}

После установления соотношений (17) приступим к вычислению вероятностей спонтанных переходов (СП) пользуясь обычными методами квантовой механики и квантовой электродинамики. В дипольном приближении связь между ЛЦ и электрическим поля задается гамильтонианом

$$
H_{\text {int }}^{e d}=-\hat{\mathbf{E}} \hat{\mathbf{d}},
$$

где $\hat{\mathbf{d}}$ есть оператор электрического дипольного момента, а $\hat{\mathbf{E}}$ есть оператор макроскопического электрического поля в месте расположения ЛЦ. В вакууме (в среде с показателем преломления равным единице)

$$
\hat{\mathbf{E}}_{\mathrm{vac}}=i \sum_{\mathbf{k}, \sigma} \sqrt{\left(2 \pi \hbar \omega_{k} / V\right)} \mathbf{e}_{\mathbf{k}, \sigma}\left[a_{\mathbf{k}, \sigma}-a_{\mathbf{k}, \sigma}^{+}\right],
$$

где $\mathbf{k}-$ волновой вектор; $\sigma$ - индекс поляризации; $a_{\mathbf{k}, \sigma}$ и $a_{\mathbf{k}, \sigma}^{+}$есть соответственно операторы уничтожения и рождения фотонов; $\mathbf{e}_{\mathbf{k}, \sigma}-$ вектор поляризации и $V$ - объем квантования. Частота фотона $\omega_{k}$ связана с волновым числом $k=|\mathbf{k}|$ дисперсионным соотношением $\omega_{k}=c k$, приводящим к плотности фотонных состояний

$$
\rho_{\mathrm{vac}}(\omega)=\omega^{2} / \pi^{2} c^{3} .
$$

В первом порядке теории возмущений скорость электродипольного (ЭД) СП из возбужденного электронного 
состояния $\mid j)$ с энергией $E_{j}$ в электронное состояния $\left.\mid j^{\prime}\right)$ с энергией $E_{j^{\prime}}$ может быть записана как [30]

$$
W_{j j^{\prime}}=\left(1 / \hbar^{2}\right) \int_{-\infty}^{+\infty} \exp (i \omega t) d_{\alpha} d_{\beta}^{*}\left\langle\hat{E}_{\alpha}(t) \hat{E}_{\beta}^{+}\right\rangle d t .
$$

Здесь $\omega=\left(E_{j}-E_{j^{\prime}}\right) / \hbar, d_{\alpha}=\left(j\left|\hat{d}_{\alpha}\right| j^{\prime}\right)(\alpha=x, y, z)$ есть матричные элементы оператора ЭД момента $\hat{\mathbf{d}}$ между состояниями $\mid j)$ и $\left.\mid j^{\prime}\right)$;

$$
\hat{E}_{\alpha}(t)=\exp \left[i H_{\mathrm{ph}} t / \hbar\right] \hat{E}_{\alpha} \exp \left[-i H_{\mathrm{ph}} t / \hbar\right],
$$

где $H_{\mathrm{ph}}=\sum_{\mathbf{k}, \sigma} \hbar \omega_{k}\left(a_{\mathbf{k}, \sigma}^{+} a_{\mathbf{k}, \sigma}+1 / 2\right)$ есть гамильтониан фотонного поля; в корреляционной функции $\left\langle\hat{E}_{\alpha}(t) \hat{E}_{\beta}^{+}\right\rangle$ символом $\langle\ldots\rangle$ обозначено усреднение с матрицей плотности фотонного поля $\hat{\rho}$ [30]. Подставляя в (21) выражение (19) для оператора $\hat{\mathbf{E}}_{\text {vac }}$ и проводя в корреляторе $\left\langle\hat{E}_{\alpha}(t) \hat{E}_{\beta}^{+}\right\rangle$усреднение с матрицей плотности $\hat{\rho}=$ $=\left|n_{\mathbf{k}_{1} \sigma_{1}}, n_{\mathbf{k}_{2} \sigma_{2}}, \ldots, n_{\mathbf{k}_{j} \sigma_{j}}, \ldots\right\rangle\left\langle n_{\mathbf{k}_{1} \sigma_{1}}, n_{\mathbf{k}_{2} \sigma_{2}}, \ldots, n_{\mathbf{k}_{j} \sigma_{j}}, \ldots\right|$, в которой $\mid n_{\mathbf{k}_{1} \sigma_{1}}, n_{\mathbf{k}_{2} \sigma_{2}}, \ldots, n_{\mathbf{k}_{j} \sigma_{j}}, \ldots$ есть фоковские состояния, находим

$$
W=(2 \pi)^{2} \sum_{\mathbf{k}, \sigma}\left(\omega_{k} / \hbar V\right)\left|\mathbf{d} \mathbf{e}_{\mathbf{k}, \sigma}\right|^{2}\left[\left\langle n_{\mathbf{k}, \sigma}^{p h}\right\rangle+1\right] \delta\left(\omega-\omega_{k}\right) .
$$

В (22) $\left\langle n_{\mathbf{k}, \sigma}^{p h}\right\rangle$ есть число фотонов в моде $(\mathbf{k}, \sigma)$. Вероятность перехода разбивается, в соответствии с постулатами Эйнштейна, на сумму

$$
W=W^{\mathrm{em}}+A_{0},
$$

где

$$
W^{\mathrm{em}}=(2 \pi)^{2} \sum_{\mathbf{k}, \sigma}\left(\omega_{k} / \hbar V\right)\left|\mathbf{d} \mathbf{e}_{\mathbf{k}, \sigma}\right|^{2}\left[\left\langle n_{\mathbf{k}, \sigma}^{p h}\right\rangle \delta\left(\omega-\omega_{k}\right)\right.
$$

есть вероятность вынужденного ЭД-излучения, а

$$
A_{0}=(2 \pi)^{2} \sum_{\mathbf{k}, \sigma}\left(\omega_{k} / \hbar V\right)\left|\mathbf{d} \mathbf{e}_{\mathbf{k}, \sigma}\right|^{2} \delta\left(\omega-\omega_{k}\right)
$$

есть вероятность спонтанного ЭД-излучения.

Усредненное по всем ориентациям векторов поляризации значение величины $\left|\mathbf{d e}_{\mathbf{k}, \sigma}\right|^{2}$ равно $|\mathbf{d}|^{2}=\left(\left|d_{x}\right|^{2}+\left.d_{y}\right|^{2}+\left.d_{z}\right|^{2}\right) / 3$. С учетом закона дисперсии $\omega_{k}=c k$ это приводит к известной формуле, впервые полученной Дираком в 1927 г. [17],

$$
A_{0}=\left(1 / \tau_{0}\right)=\left(4 \pi^{2} \omega|\mathbf{d}|^{2} / 3 \hbar\right) \rho_{\mathrm{vac}}(\omega),
$$

Оператор макроскопического электрического поля в объемной среде в дипольном приближении есть

$$
\hat{\mathbf{E}}=i \sum_{\mathbf{k}, \sigma}\left(2 \pi \hbar \omega_{k} / V \varepsilon\right)^{1 / 2} \mathbf{e}_{\mathbf{k}, \sigma}\left[a_{\mathbf{k}, \sigma}-a_{\mathbf{k}, \sigma}^{+}\right],
$$

где $\varepsilon=n^{2}$ - диэлектрическая проницаемость среды. Дисперсионное соотношение теперь есть $\omega_{k}=c k / n$, что приводит к изменению плотности фотонных состояний:

$$
\rho_{\text {bulk }}(\omega)=n^{3} \rho_{\text {vac }}(\omega) \text {. }
$$

В результате получаем известную формулу [31]

$$
A_{\text {bulk }}=1 / \tau_{\text {bulk }}=n A_{0}
$$

Для учета отличия макроскопического значения напряженности электрического поля в месте расположения ЛЦ $\left(E_{\text {macro }}\right)$ от действующего (микроскопического) поля $\left(E_{\text {loc }}\right)$ в формулу $(29$ a) вводят $[32,33]$ множитель $f_{L}=\left(E_{\mathrm{loc}} / E_{\mathrm{macro}}\right)^{2}$ (так называемая поправка на локальное поле). С учетом этой поправки формула (29a) приобретает вид

$$
A_{\text {bulk }}=1 / \tau_{\text {bulk }}=n f_{L}(n) A_{0}
$$

Во всех существующих моделях локального поля $f_{L}$ есть функция показателя преломления среды $n$, при этом $f_{L}(1)=1$. Таким образом, мы видим, что помимо поправки на локальное поле $f_{L}$, две главные физические причины обуславливают изменение скорости СП в ЛЦ при перемещении его из вакуума в объемную диэлектрическую среду [27]. Во-первых, как видно из уравнения (28), меняется плотность фотонных состояний. Во-вторых, как видно из сравнения формул (19) и (27), меняется амплитуда нулевых колебаний электрического поля, ответственных за спонтанный распад. А именно, отношение их квадратов есть $\left(E / E_{\mathrm{vac}}\right)^{2}=1 / \varepsilon=1 / n^{2}$. Вместе эти две причины приводят в кристалле к фактору $\left(1 / n^{2}\right) n^{3}=n$ в отношении

$$
A_{\text {bulk }} / A_{0}=n f_{L}(n)
$$

Как следует из формул (16) и (27), в рассматриваемом нами случае операторы электрического поля будут иметь вид

$$
\hat{\mathbf{E}}_{s}=i g_{s} \sum_{\mathbf{k}, \sigma}\left(2 \pi \hbar \omega_{k} / V \varepsilon_{\mathrm{eff}}\right)^{1 / 2} \mathbf{e}_{\mathbf{k}, \sigma}\left[a_{\mathbf{k}, \sigma}-a_{\mathbf{k}, \sigma}^{+}\right]
$$

Таким образом, общее выражение для скоростей ЭДпереходов в матрице и НЧ может быть записано как

$$
A_{s}=1 / \tau_{0}^{(s)}=n_{\mathrm{eff}} g_{s}^{2} f_{L}\left(n_{s}\right) A_{0}^{(s)}
$$

где $g_{s}$ задаются формулами (17), а показатели преломления $n_{s}=\sqrt{\varepsilon}_{s}$ (напомним, что индекс $s$ пробегает значения 1, 2, 3 для ЛЦ, расположенных в матрице, оболочке и ядре соответственно). В формуле (31) $A_{0}^{(s)}$ задается выражением

$$
A_{0}^{(s)}=\left(4 \pi^{2} \omega_{s}\left|\mathbf{d}^{(s)}\right|^{2} / 3 \hbar\right) \rho_{\mathrm{vac}}\left(\omega_{s}\right)
$$

в котором индекс $s$ указывает, что в принципе частота и дипольный момент заданного перехода одного и того же ЛЦ могут меняться при помещении ЛЦ в матрицу, оболочку или ядро. 


\section{5. Скорости спонтанных излучательных переходов в матрице, оболочке и ядре}

Полагая в уравнении (32) $s=1$, находим, что скорость СП в ЛЦ, находящегося в матрице, равна

$$
A_{\text {matr }}=n_{\text {eff }} f_{L}\left(n_{1}\right) A_{0}^{(1)} /(1-f \beta)^{2} .
$$

Аналогично, полагая $s=2$, находим для ЛЦ в оболочке

$$
A_{\text {shell }}=n_{\text {eff }}\left[3 \varepsilon_{1}\left(\varepsilon_{3}+2 \varepsilon_{2}\right) /(1-f \beta) \Delta\right]^{2} f_{L}\left(n_{2}\right) A_{0}^{(2)}
$$

и для ЛЦ в ядре $(s=3)$

$$
A_{\text {core }}=n_{\text {eff }}\left[9 \varepsilon_{1} \varepsilon_{2} /(1-f \beta) \Delta\right]^{2} f_{L}\left(n_{3}\right) A_{0}^{(3)} .
$$

Значение $f=0$ соответствует изолированной частице и формулы (33)-(35) переходят в соответствующие формулы работы [30]. (В работе [30] были получены аналитические выражения для скоростей СП в ЛЦ, расположенных внутри и вне изолированной субволновой слоистой сферической НЧ, погруженной в диэлектрическую матрицу). Чтобы выявить масштабность обсуждаемого механизма изменения скоростей СП при перемещении ЛЦ из объемного материала в различные места нанокомпозита, сравним $A_{\text {matr, }}, A_{\text {shell }}$ и $A_{\text {core }}$ (см. формулы (33)-(35)) со значениями скоростей СП $\left(A_{\text {bulk }}^{\text {matr }}\right.$, $A_{\text {bulk }}^{\text {shell }}$ и $\left.A_{\text {bulk }}^{\text {core }}\right)$ в соответствующем объемном материале такого же состава и структуры. При этом будем далее полагать, во-первых, что модули дипольных моментов перехода $|\mathbf{d}|$ не меняются. Во-вторых, что поправка на локальное поле $f_{L}$ одинакова в составных частях НЧ и в объемном материале из которых эти части приготовлены. Тогда отношение соответствующих скоростей дается формулами

$$
\begin{gathered}
A_{\text {matr }} / A_{\text {bulk }}^{\text {matr }}=n_{\mathrm{eff}} / n_{1}(1-f \beta)^{2}, \\
A_{\text {shell }} / A_{\text {bulk }}^{\text {shell }}=n_{\mathrm{eff}}\left[3 \varepsilon_{1}\left(\varepsilon_{3}+2 \varepsilon_{2}\right)\right]^{2} / n_{2}[(1-f \beta) \Delta]^{2}, \\
A_{\text {core }} / A_{\text {bulk }}^{\text {core }}=n_{\mathrm{eff}}\left[9 \varepsilon_{1} \varepsilon_{2}\right]^{2} / n_{3}[(1-x \beta) \Delta]^{2} .
\end{gathered}
$$

\section{6. Обсуждение}

Если в предыдущих формулах положить $\varepsilon_{2}=\varepsilon_{3}$ или $\xi=(b / a)^{3}=0$, мы перейдем к рассмотрению нанокомпозита с включениями из однородных сферических НЧ. Тогда для ЛЦ в матрице мы получаем формулу того же вида, что и (33), а именно:

$$
A_{\text {matr }}=n_{\text {efff }} f_{L}\left(n_{1}\right) A_{0} /(1-f \beta)^{2},
$$

но в которой теперь

$$
\beta=\left(\varepsilon_{2}-\varepsilon_{1}\right) /\left(\varepsilon_{2}+2 \varepsilon_{1}\right),
$$

a $n_{\text {eff }}$ определяется формулой Максвелла Гарнетта (4). Для ЛЦ внутри НЧ получаем

$$
A_{\text {nano }}=n_{\text {eff }}\left[3 \varepsilon_{1} /\left[2 \varepsilon_{1}+\varepsilon_{2}-f\left(\varepsilon_{2}-\varepsilon_{1}\right)\right]\right]^{2} f_{L}\left(n_{2}\right) A_{0} .
$$

Это выражение совпадает с ранее найденной формулой в работе [34]. Отношение скорости СП для ЛЦ внутри НЧ $\left(A_{\text {nano }}\right)$ к скорости СП в объемном материале такого же состава и структуры $\left(A_{\text {bulk }}\right)$ при оговоренных выше условиях (одинаковость модулей дипольных моментов перехода $|\mathbf{d}|$ и поправок на локальное поле $f_{L}$ ) дается формулой:

$$
A_{\text {nano }} / A_{\text {bulk }}=\left(n_{\text {eff }} / n_{2}\right)\left[3 \varepsilon_{1} /\left[2 \varepsilon_{1}+\varepsilon_{2}-f\left(\varepsilon_{2}-\varepsilon_{1}\right]\right]^{2} .\right.
$$

Сравнение формулы (41 b) с экспериментальными данными дано в работе [35]. Для изолированной НЧ формулы (41a) и (41 b) переходят соответственно в формулы:

$$
\begin{aligned}
& A_{\text {nano }}=n_{1}\left[\left(3 \varepsilon_{1}\right) /\left(2 \varepsilon_{1}+\varepsilon_{2}\right)\right]^{2} f_{L}\left(n_{2}\right) A_{0}, \\
& A_{\text {nano }} / A_{\text {bulk }}=\left(n_{1} / n_{2}\right)\left[3 \varepsilon_{1} /\left(2 \varepsilon_{1}+\varepsilon_{2}\right)\right]^{2} .
\end{aligned}
$$

Выражение (42b) совпадает с результатом работы [36], а также с аналитическим результатом работы [37]. Отметим, что (42b) определяет отношение $A_{\text {nano }} / A_{\text {bulk }}$ справедливое, при оговоренных выше условиях, для всех функциональных зависимостей $f_{L}(n)$. Интересно отметить, что для НЧ, взвешенной в среде с $\varepsilon_{1}=1$, из (42 а) следует, что $A_{\text {nano }}=A_{0}$, если $f_{L}(n)$ есть поправка на локальное поле в модели Лорентца, для которой $f_{L}(n)=\left[\left(n^{2}+2\right) / 3\right]^{2}[38]$.

Другой подход к проблеме времени жизни ЛЦ в НЧ, находящейся в составе нанокомпозита с включениями однородных сферических НЧ $\left(\tau_{\text {nano }}\right)$, был принят в упомянутой выше работе [14]. Авторы исходили из формулы для времени жизни возбужденных состояний ЛЦ при ЭД переходе для ЛЦ в объемной диэлектрической среде (см. формулу (1) в [14]):

$$
\tau_{\text {bulk }} \sim \frac{1}{f(E D)} \frac{\lambda_{0}^{2}}{\left[\left(n^{2}+2\right) / 3\right]^{2}},
$$

где $f(E D)$ есть сила осциллятора для ЭД перехода, $\lambda_{0}$ - длина волны в вакууме и $\mathrm{n}$ есть показатель преломления среды. Выражение для $\tau_{\text {nano }}$ для ЛЦ в нанокристаллах такого же состава и той же структуры, что и объемный кристалл, авторы работы [14] получают модификацией выражения (43) заменой в нем $n$ на $n_{\text {eff. }}$ Такая замена аргументируется (i) изменением фотонной плотности состояний и (ii) модификацией поляризуемости среды [14]. Первый фактор (i), как источник изменения скорости СП, начал учитываться со времени появления в 1946 г. работы Парселла [28] и не вызывает возражений. Авторы, однако, не разъясняют, как из второго фактора (ii) следует необходимость замены $n$ 


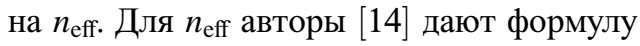

$$
n_{\mathrm{eff}}=f n_{1}+(1-f) n_{2}
$$

где $n_{1}$ и $n_{2}$ есть соответственно показатели преломления среды и материала, из которого приготовлены НЧ. Отметим, что формула (44) принадлежит к широко используемому классу моделей эффективной среды, сформированному так называемыми „степенными“ приближениями [24]:

$$
\varepsilon_{\mathrm{eff}}^{\gamma}=f \varepsilon_{1}^{\gamma}+(1-f) \varepsilon_{2}^{\gamma}
$$

Принцип „степенных“ приближений прост: некоторая степень диэлектрических проницаемостей среды и включений усредняется с весами, пропорциональными их объемным долям [24]. Степень $\gamma=1 / 2$ соответствует уравнению (44) и была предложена в работе [39]. Предлагались также степени $\gamma=1 / 3$ [40] и $\gamma=1$ [41]. Отметим, что зависимость (45) с $\gamma=1 / 3$ получена также в курсе Ландау и Лифшица (см. §9 в [29]), но не исходя из изложенного выше принципа, а выведена в приближении $\left|\varepsilon_{1}-\varepsilon_{2}\right| \ll \varepsilon_{1}, \varepsilon_{2}$. Там же $(\S 9$ в [29]) рассмотрен ,другой предельный случай, допускающий точное рассмотрение, - диэлектрическая проницаемость эмульсии с произвольной разницей между диэлектрическими проницаемостями среды $\left(\varepsilon_{1}\right)$ и диспергированной фазы $\left(\varepsilon_{2}\right)$, но малой концентрацией последней; частицы диспергированной фазы предполагаются сферическими.“. Соответствующее выражение (формула в (9.7) в [29]) есть

$$
\varepsilon_{\mathrm{eff}}=\varepsilon_{1}+f 3 \varepsilon_{1}\left(\varepsilon_{2}-\varepsilon_{1}\right) /\left(\varepsilon_{2}+2 \varepsilon_{1}\right) .
$$

Как видно из формулы Максвелла Гарнетта (4), формула (46) следует из (4) при малых значениях концентрации $f$. Работа [14] привлекла большое внимание к проблеме спонтанного излучения ЛЦ в нанокристаллах и стимулировала дальнейшие ее исследования. Формула

$$
A_{\text {nano }}=1 / \tau_{\text {nano }}=n_{\text {eff }} f_{L}\left(n_{\text {eff }}\right) A_{0},
$$

полученная из формулы (29b) заменой $n \rightarrow n_{\text {eff }}$ используется в целом ряде работ (см., например, обзоры [1-4] и ссылки в них). Из формул (29 b) и (47) следует, что значение отношения $A_{\text {nano }} / A_{\text {bulk }}$, равно

$$
A_{\text {nano }} / A_{\text {bulk }}=\left(n_{\mathrm{eff}} / n\right)\left[f_{L}\left(n_{\mathrm{eff}}\right) / f_{L}(n)\right]
$$

и существенно зависит от выбора той или иной модели локального поля. Это обстоятельство инициировало работы по определению модели локального поля (детальное обсуждение этой проблемы дано в обзоре [3]). В оригинальной работе [14] в качестве поправки бралась поправка $\left[\left(n^{2}+2\right) / 3\right]^{2}$ в модели Лорентца [38]. (Эту модель в современной литературе, посвященной излагаемой тематике, часто называют моделью виртуальной полости (virtual-cavity model) [3].) В таком случае для НЧ, взвешенной в среде с $\varepsilon_{1}=1$, из (47) следует, что $A_{\text {nano }}=A_{0}$. Как было отмечено выше, тот же результат дает формула (42a). Т.е., в этом случае оба рассмотренных подхода дают одинаковый результат: $A_{\text {nano }}=1 / \tau_{\text {nano }}=A_{0}$. Кстати, этот результат содержится уже в неявной форме в известной книге Киттеля [38], где показано, что в статическом приближении локальное (действующее) поле в сферической частице, помещенной в вакуум, равно нулю (см. [38], глава 13).

Давно известно, что редкоземельные ЛЦ являются хорошими зондами локальных полей в неоднородных средах и вышеизложенное является еще одним подтверждением этого факта. Здесь не рассмотрено, однако, влияние на СП ближних полей, создаваемых дипольными моментами $\mathbf{p}_{1}=\alpha_{1} \mathbf{E}_{1}$ и $\mathbf{p}_{2}=\alpha_{2} \mathbf{E}_{1}$ (см. формулы $(3 \mathrm{~b})$ и (3 a) соответственно). Для изолированных сферических НЧ-структуры „ядро-оболочка“ это влияние учтено в работе [42]. (Аналогичную проблему для активированных нанокомпозитов предполагается рассмотреть в будущем.) Как показано в работе [43], влияние ближнего поля особенно сильно будет сказываться на сверхчувствительных переходах в Р3-ионах.

\section{7. Заключение}

Полученные аналитические выражения для скоростей спонтанного ЭД-распада возбуждения ЛЦ, находящихся в субволновых сферических НЧ-структуры „ядро-оболочка“" и в матрице нанокомпозита, являются несложными рациональными функциями от диэлектрических проницаемостей матрицы $\left(\varepsilon_{1}\right)$, оболочки $\left(\varepsilon_{2}\right)$, ядpa $\left(\varepsilon_{3}\right), \varepsilon_{\text {eff }}$, фактора заполнения $(f)$ и отношения объема ядра НЧ к объему всей НЧ. Это открывает конструктивный путь целенаправленного выбора параметров оболочки и ядра слоистых частиц для получения желаемых излучательных характеристик ЛЦ в нанокомпозите.

\section{Список литературы}

[1] Xueyuan Chen, Yongsheng Liu, Datao Tu. Lanthanidedoped luminescent nanomaterials. From fundamentals to bioapplications. Springer-Verlag, Berlin (2014).

[2] P.A. Tanner. In: Lanthanide Luminescence Photophysical. Analytical and Biological Aspects / Eds P. Hänninen, H. Härma. Springer, Heidelberg, N. Y. (2011). P. 183.

[3] K. Dolgaleva, R.W. Boyd. Adv. Opt. Photon. 4, 11 (2012).

[4] Yongsheng Liu, Datao Tu, Haomiao Zhu, Xueyuan Chen. Chem. Soc. Rev. 42, 12, 6924 (2013).

[5] В.В. Овсянкин, П.П. Феофилов. Письма в ЖЭТФ 3, 12, 322 (1966).

6] F. Auzel, C.R. Acad. Sci. 262, 1016 (1966).

[7] Т.Т. Басиев, М.Е. Дорошенко, В.В. Осико. Письма в ЖЭТФ 71, 1,14 (2000).

[8] F. Auzel. Chem. Rev. 104, 1139 (2004).

[9] H. Schäfer, M. Haase. Angew. Chem. Int. Ed. 50, 5808 (2011).

[10] Chenglin Yan , Haiguang Zhao, D.F. Perepichka, F. Rosei. Small, 12, 29, 3888 (2016).

[11] Т.Т. Басиев, Ю.В. Орловский, К.К. Пухов, П.П. Федоров, Оптический лазерный материал и способ его применения. Патент 2428778 РФ (29.05.2009). 
[12] Y. Tzuk, C. Goren, D. Raanan, G. Strum. Opt. Lett. 37, 5, 939 (2012).

[13] Thermometry at the Nanoscale: Techniques and Selected Applications. Series: RSC Nanoscience \& Nanotechnology No. 38 (The Royal Society of Chemistry). / Eds L.D. Carlos, F. Palacio Thomas Graham House, Cambridge, UK (2016).

[14] R.S. Meltzer, S.P. Feofilov, B. Tissue, H.B. Yuan. Phys. Rev. B 60, 20 R14012 (1999).

[15] T. Sun, F. Wang. In: Phosphors, Up Conversion Nano Particles, Quantum Dots and their Applications / Ed. RuShi Liu. Springer, Singapore (2016). V. 2. P. 507.

[16] Qian Liu, Yunxiang Zhang, Chunte Sam Peng, Tianshe Yang, L.-M. Joubert, S. Chu. Nature Photon. 12, 548 (2018).

[17] П.А.М. Дирак. Принципы квантовой механики. Физматлит, M. (1960).

[18] A.L. Aden, M. Kerker. J. Appl. Phys. 22, 10, 1242 (1951).

[19] A.E. Neeves, M.H. Birnboim. J. Opt. Soc. Am. B 6, 4787 (1989).

[20] J.C. Maxwell Garnett. Philos. Trans. R. Soc. London A 203, 385 (1904).

[21] J.C. Maxwell Garnett. Philos. Trans. R. Soc. London A 205, 237 (1906).

[22] C.F. Bohren, D.R. Huffman. Absorption and scattering of light by small particles. John Wiley, N. Y., (1983).

[23] Tuck C. Choy. Effective Medium Theory: Principles and Applications. 2-nd ed. Oxford University Press, Oxford, (2016).

[24] A. Sihvola. Electromagnetic Mixing Formulas and Applications. The Institution of Electrical Engineers. N, Y. (1999).

[25] М. Борн, Э. Вольф. Основы оптики. Наука, М. (1973).

[26] A. Sihvola, I.V. Lindell. J. Electromagn. Waves Appl. 2, 8, 741 (1988).

[27] K.K. Pukhov, T.T. Basiev. Opt. Mater. 32, 12, 1664 (2010).

[28] E.M. Purcell. Phys. Rev. 69, 11-12, 681 (1946).

[29] Л.Д. Ландау, Е.М. Лифшиц. Электродинамика сплошных сред. Наука, М. (1982).

[30] K.K. Pukhov. Opt. Mater. 74, 12, 166 (2017).

[31] G. Nienhuis, C.Th.J. Alkemade. Physica (Amsterdam) C81, 1, 181 (1976).

[32] M. Lax. J. Chem. Phys. 20, 11, 1752 (1952).

[33] B. Henderson, G.F. Imbusch. Optical spectroscopy of inorganic solids. Clarendon Press, Oxford (1989).

[34] К.К. Пухов, Т.Т. Басиев, Ю.В. Орловский. Письма в ЖЭТФ 88, 1, 14 (2008).

[35] Yanhua Wang, Yongsheng Liu, Qingbo Xiao, Haomiao Zhu, Renfu Li, Xueyuan Chen. Nanoscale 3, 3164 (2011).

[36] E. Yablonovitch, T.J. Gmitter, R. Bhat. Phys. Rev. Lett. 61, 22, 2546 (1988).

[37] H. Chew. Phys. Rev. A 38, 7, 3410 (1988).

[38] Ч. Киттель. Введение в физику твердого тела. Наука, M. (1978).

[39] J.R. Birchak, L.G. Gardner, J.W. Hipp, J.M. Victor. Proc. IEEE 62, 1, 93 (1974).

[40] H. Looyenga. Physica 31, 3, 401 (1965).

[41] F.T. Ulaby, R.K. Moore, A.K. Fung. Microwave remote sensing - Active and passive. Artech House, Norwood, Mass. (1986). V. 3

[42] K.K. Pukhov. J. Lumin. 169, Part B (Special Issue), 503 (2016).

[43] К.К. Пухов, С.К. Секацкий. Оптика и спектроскопия, 116, 5770 (2014).

Редактор Ю.Э. Китаев 